\section{Necrotising orbital cellulitis}

S.G.J. NG, R. NAZIR, C.P.K. SABHUDI,

R.D. LAITT, A. MALOOF,

B. LEATHERBARROW

\begin{abstract}
Purpose To report 2 cases of severe necrotising orbital cellulitis which illustrate the need for aggressive surgical management to prevent blindness.

Methods The case records of 2 patients with necrotising orbital cellulitis were reviewed. Results Both patients had orbital cellulitis associated with sinusitis. Each case was characterised by the rapid development of severe systemic toxicity, extensive soft tissue necrosis and abscess formation. One patient developed panophthalmitis and the eye had to be eviscerated. The other patient underwent repeated surgical drainage of multiple orbital abscesses. This led to resolution of the infection and preservation of vision. Conclusions Atypical rapidly progressive necrotising orbital cellulitis may occasionally be encountered. In such cases, aggressive surgical drainage of orbital abscesses is crucial to prevent blindness and death.
\end{abstract}

Key words Necrotising cellulitis, Necrotising fasciitis, Orbital cellulitis, Streptococcus anginosus

In orbital cellulitis medical management with intravenous antibiotics is now the mainstay of treatment. Cases of severe infection that do not respond to antibiotics can present major management difficulties. We present 2 cases of necrotising orbital cellulitis characterised by the rapid development of severe systemic toxicity, extensive orbital and periorbital soft tissue necrosis and abscess formation. Both cases involved visual morbidity and life-threatening infection.

\section{Case reports}

\section{Case 1}

A 30-year-old man presented with a $12 \mathrm{~h}$ history of pain and swelling around his left eye. He was febrile and toxic. He had had 2 weeks of 'toothache' and left cheek pain. He had a history of chronic sinusitis and numerous facial traumas. On examination he had a temperature of $38^{\circ} \mathrm{C}$. He had a left non-axial proptosis, severe periorbital oedema, chemosis and pain on eye movement. A visual acuity was not recorded. Orbital cellulitis was diagnosed. A computed tomography (CT) scan (Fig. 1) demonstrated left maxillary sinusitis with prominent superficial soft tissue swelling. This was most marked in the temporal fossa where gas was seen between tissue planes. There was proptosis with abnormal post-septal soft tissue extending along the lateral orbital wall. An old left orbital floor blow-out fracture was present.

Intravenous flucloxacillin was commenced. Blood cultures did not yield any organisms. Forty-eight hours after admission he underwent a left antral lavage with drainage of copious amounts of pus. Culture of the pus revealed a heavy growth of a $\beta$-haemolytic Group $F$ Streptococcus, anaerobic Gram-negative bacilli and a non-haemolytic non-groupable Streptococcus. On the advice of a microbiologist his antibiotics were changed to intravenous cefuroxime, metronidazole and rifampicin. Over the next $48 \mathrm{~h}$ his systemic condition deteriorated. His temperature remained high and he was obtunded. The proptosis of his left eye increased. The left side of his face and neck became more swollen. Subcutaneous surgical emphysema was present. His lower lid became necrotic (Fig. 2). No afferent pupillary defect was detected. On day 4 after admission his systemic condition began to improve but there was a purulent discharge from the left eye, the cornea became hazy and a hypopyon developed. The visual acuity was recorded as perception of light. Suppuration of the cornea increased and it perforated 2 days later (Fig. 3). He was transferred to our institution.

A full medical history and examination did not reveal any cause of immunosuppression. He underwent an evisceration along with drainage of subperiosteal, orbital, lower lid and temporalis fossa abscesses. Copious pus was drained from each site. Streptococcus anginosus was cultured from pus swabs from these sites. Histopathological examination of the ocular contents revealed a panophthalmitis. There was a polymicrobial infection consisting of Grampositive cocci and Gram-negative bacilli along with widespread purulent necrosis.
S.G.J. $\mathrm{Ng}$

R. Nazir

A. Maloof

B. Leatherbarrow

Manchester Royal Eye Hospital Manchester, UK

C.P.K. Sabhudi Microbiology Department Manchester Royal Infirmary Manchester, UK

R.D. Laitt

Neuroradiology Department Manchester Royal Infirmary Manchester, UK

Mr Brian Leatherbarrow Manchester Royal Eye Hospital

Oxford Road

Manchester M13 9WH, UK

Tel: +44(0)1612765569 Fax: +44 (0)1612726618 e-mail: bollin@mail.mightymicro.co.uk

Received: 16 May 2000 Accepted in revised form: 20 October 2000 


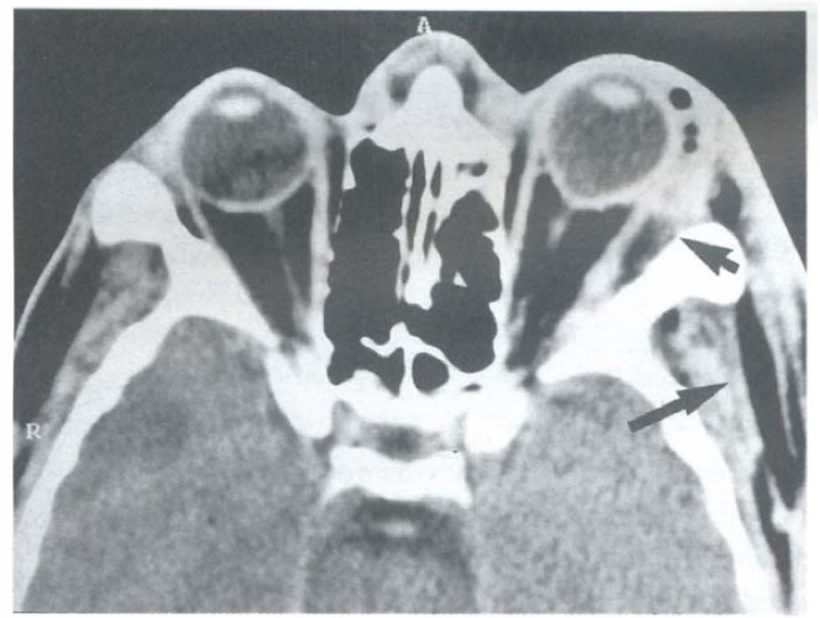

Fig. 1. Case 1. Axial CT scan through the left orbit demonstrating proptosis with traction on the optic nerve and associated distortion of the globe. Abnormal soft tissue is seen extending along the lateral orbital wall (small arrow) with prominent soft tissue swelling in the temporal fossa (long arrow). The tissue planes are separated by gas.

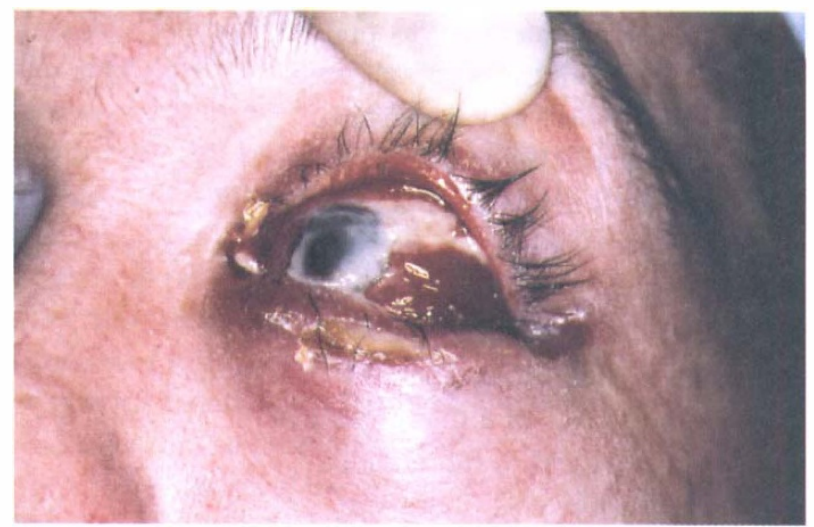

Fig. 3. Case 1. Day 7: necrotic anterior segment tissues and corneal perforation.

\section{Case 2}

A 13-year-old boy presented to a children's hospital with a $12 \mathrm{~h}$ history of pain and swelling of his left eye. He had a 2 day history of cough and vomiting. On examination he was alert, conscious and orientated; his temperature was $37.4^{\circ} \mathrm{C}$. He had marked periorbital oedema, a left non-axial proptosis, chemosis and painful limitation of his eye movements. The visual acuity was not recorded. His cornea was clear and there was no relative afferent pupillary defect. Orbital cellulitis was diagnosed and intravenous cefotaxime and rifampicin were commenced. A CT scan (Fig. 4) demonstrated extensive left periorbital soft tissue swelling, proptosis and left maxillary and ethmoidal sinusitis. There was associated superficial soft tissue swelling with gas separating tissue planes in the temporal fossa. Gas containing subperiosteal soft tissue was also seen extending along the medial orbital wall. These changes were consistent with a subperiosteal abscess. Over the next $6 \mathrm{~h}$ his systemic condition deteriorated. The left orbit became progressively tenser, the proptosis increased, there was marked resistance to retropulsion with $3 \mathrm{~mm}$ of lagophthalmos.

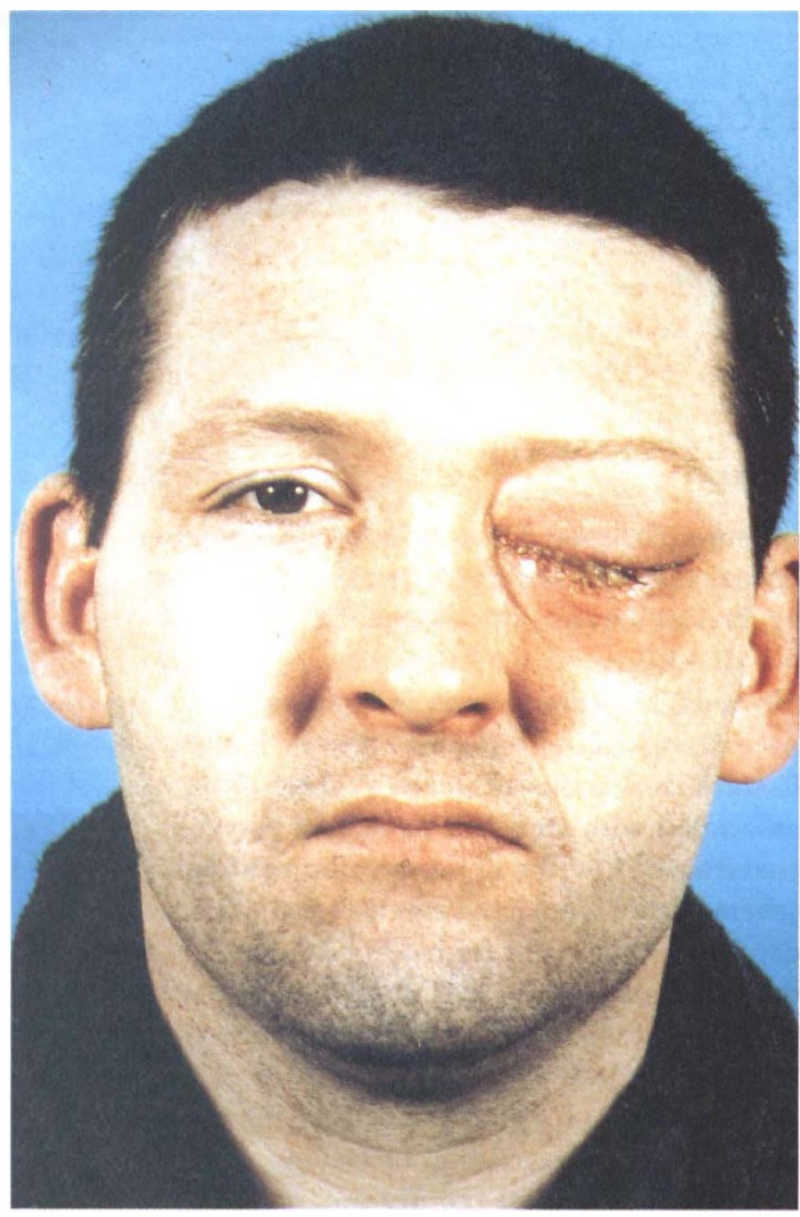

Fig. 2. Case 1. Day 7: left periorbital swelling and necrotic lower lid.

He was transferred to our institution and underwent emergency drainage of a subperiosteal abscess along with maxillary and ethmoidal sinus drainage via a Lynch incision over the medial orbital rim. At the end of the procedure a gutter drain was placed in the wound. Intravenous metronidazole was added to his medications. Twenty-four hours later his condition had not improved. He remained febrile and toxic. The affected eye's visual acuity was counting fingers. There was little discharge from his drain. Frequent ocular lubrication was applied for the severe lagophthalmos and corneal exposure. A pus swab grew Streptococcus anginosus. A blood culture was negative. After consultation with a microbiologist intravenous benzylpenicillin and chloramphenicol replaced his previous antibiotic regime. A full paediatric investigation did not reveal any evidence of immunosuppression that might have predisposed him to severe infection. A repeat CT scan showed left maxillary, ethmoidal and sphenoidal sinusitis and diffuse orbital soft tissue oedema.

He then underwent an anterior orbital drainage and a formal anterior ethmoidectomy in conjunction with an ENT surgeon. Seventy-two hours after admission his condition had still not improved significantly. A third CT scan (Fig. 5) demonstrated abnormal gas-containing areas of soft tissue behind the globe consistent with orbital abscesses. He underwent a further orbital exploration resulting in the drainage of large superior 


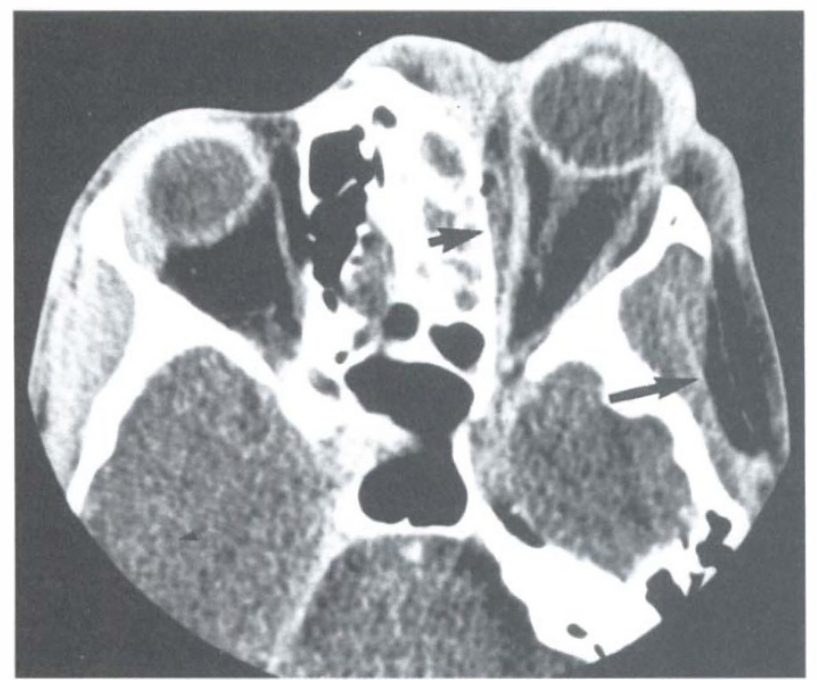

Fig. 4. Case 2. CT scan on presentation demonstrating proptosis and subperiosteal abscess along the medial orbital wall (short arrow). Superficial soft tissue swelling is also seen in the temporal fossa with gas separating soft tissue planes (long arrow).

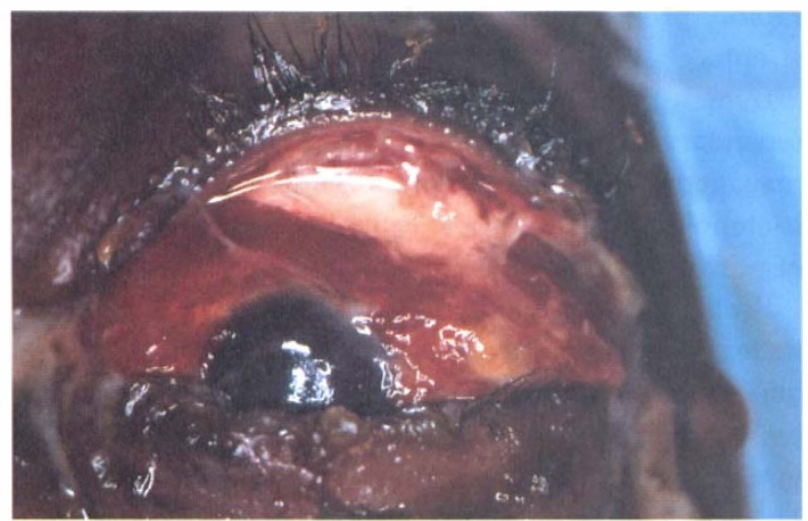

Fig. 6. Case 2. Day 3: persistent superior orbital abscesses discharging into the superior fornix despite two previous orbital drainage operations.

orbital abscesses (Fig. 6). Further pus swabs were obtained at each operation. Microbiological examination of each of the pus swabs demonstrated a monomicrobial infection of Streptococcus anginosus. Anaerobes were sought but not isolated in any of the samples. Following his third operation he began showing steady systemic and orbital improvement. His drains were removed 5 days post-operatively and he was changed to oral antibiotics. Ten days after discharge his visual acuity in his left eye was 6/24 unaided and he had a moderate mechanical ptosis. Five months later his visual acuity in his left eye had improved to $6 / 9$, the ptosis had almost completely resolved and he had a small divergent squint (Fig. 7).

\section{Discussion}

The precise roles of medical and surgical treatment in orbital cellulitis are debated. The disease is commonest in children and the most frequent cause is direct extension of paranasal sinusitis. In children the prompt use of appropriate intravenous antibiotics can lead to resolution of infection even where there is radiological evidence of a subperiosteal abscess. ${ }^{1,2}$ As it may be difficult to

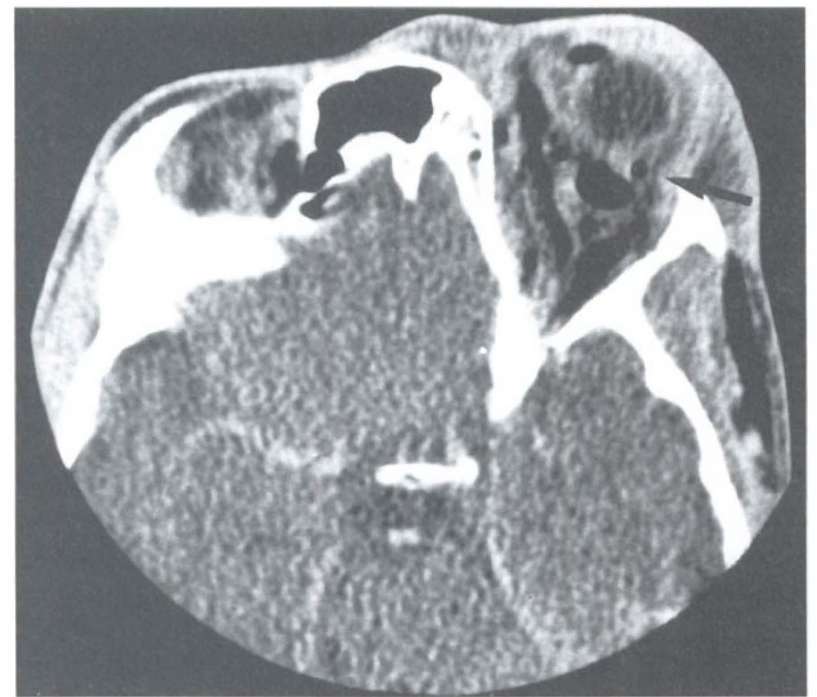

Fig. 5. Case 2. Day 3: CT scan demonstrating retrobulbar gas containing soft tissue consistent with orbital abscess (arrow).

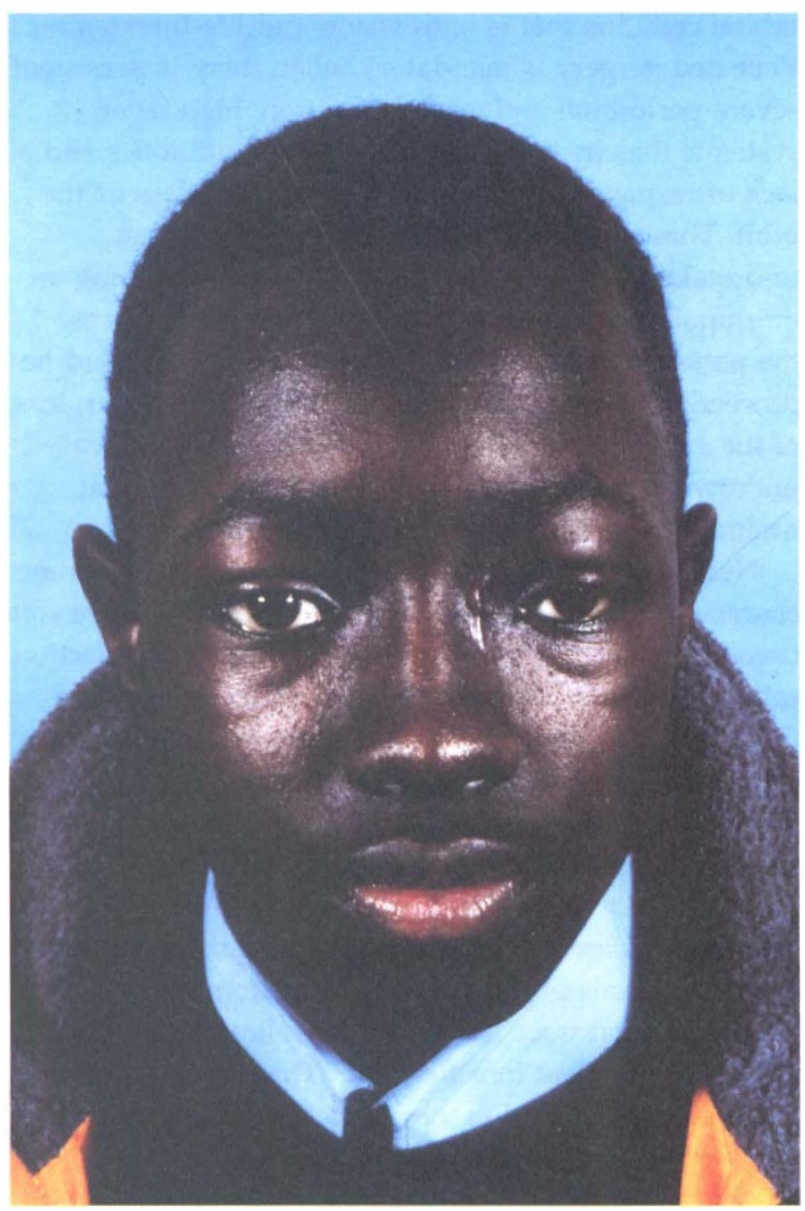

Fig. 7. Case 2. Five months after discharge: resolved ptosis, left divergent squint and small scar on the side of the nose.

distinguish radiologically between a subperiosteal abscess and inflammatory swelling, CT scan appearances should be treated as adjunctive information only. A common approach is to use a trial of high-dose intravenous antibiotics and to consider surgical drainage if there is failure to respond rapidly. ${ }^{1-4}$ Where medical treatment fails, direct drainage via an external skin incision is the conventional surgical approach. More recently endonasal endoscopic surgery has been 


\begin{tabular}{|c|c|c|}
\hline & Necrotising orbital cellulitis & Orbital necrotising fasciitis ${ }^{19-21}$ \\
\hline Causative organisms: & Mixed aerobes/anaerobes & Group A $\beta$-haemolytic Streptococcus \\
\hline Common predisposing causes: & Sinusitis & $\begin{array}{l}\text { Trauma, surgery, upper respiratory tract } \\
\text { infection, immunosuppression }\end{array}$ \\
\hline Clinical findings: & Extensive cellulitis and abscess formation & Purplish discoloration of skin \\
\hline Cause of visual loss: & Panophthalmitis & Retinal and choroidal ischaemia \\
\hline CT scan: & \pm gas in soft tissues & Non-gas-forming \\
\hline Treatment: & Aggressive surgical debridement & $\begin{array}{l}\text { Aggressive surgical debridement; life } \\
\text { support may be required }\end{array}$ \\
\hline
\end{tabular}

advocated. ${ }^{5}$ In adults sinusitis is also the most common cause of orbital cellulitis and it is treated in an identical manner to children. ${ }^{6-8}$ Less common causes of orbital cellulitis are penetrating injury, lid disease, lacrimal disease and ocular surgery. Rarer causes are peribulbar injection, ${ }^{9}$ endophthalmitis ${ }^{10}$ and endogenous infection. $^{11}$

Our 2 case histories represent a fulminant form of orbital cellulitis that is both vision and life-threatening. Repeated surgery is mandatory when there is persistent severe periorbital and orbital infection, high fever, systemic toxicity, a lack of response to antibiotics and a lack of response to surgical drainage of sinuses or the orbit. These 2 cases illustrate that surgery is best undertaken by a multidisciplinary team comprising an otorhinolaryngologist and an orbital surgeon. In case 1 the patient did not improve after antral lavage. Had he proceeded to an orbital drainage procedure sooner, loss of the eye may have been prevented. In contrast, case 2 underwent prompt, repeated orbital and ethmoidal drainage procedures that probably saved the eye.

Necrotising cellulitis is a form of microbial gangrene characterised by the rapid development of extensive soft tissue necrosis. ${ }^{12}$ It is typically a polymicrobial infection caused by aerobic and anaerobic pathogens or mixed Clostridia. In animal studies a mixture of organisms can cause more severe infection than each of the organisms singly (bacterial synergy). Necrotising cellulitis can be crepitant (gas-forming) or non-crepitant.

Streptococcus anginosus was isolated in both our cases. This is a species of the Streptococcus milleri group, which are commensals of the mouth, nasopharynx and gastrointestinal tract. They have a well-established role in serious purulent infections. ${ }^{13,14}$ Their propensity to cause abscess formation may be related to a polysaccharide capsule that hinders phagocytosis, the production of hydrolytic enzymes or the presence of capsular proteins that suppress lymphocyte and fibroblast proliferation but do not affect leucocyte migration. ${ }^{15}$ Most Streptococcus anginosus infections follow local invasion from a site where these organisms are part of the normal flora.

In case 1 there was a mixed infection and bacterial synergy may have contributed to the severity of infection. In case 2 the necrotising orbital cellulitis appears to have been caused by Streptococcus anginosus alone since no anaerobes were isolated from several different pus swabs. Another unusual feature in case 2 was the gas formed by the infection. While crepitant infection is usually associated with anaerobic infection, Streptococcus anginosus has previously been reported to cause gas-forming soft tissue infection. ${ }^{16}$ Since streptococci are now the commonest pathogen associated with orbital cellulitis, ${ }^{17,18}$ in the future we may see a greater incidence of atypical orbital cellulitis caused by virulent streptococci such as Streptococcus anginosus.

These 2 cases of necrotising cellulitis have many similarities to necrotising fasciitis (NF), which is another rapidly progressive subcutaneous life-threatening streptococcal infection. Orbital cellulitis in NF is extremely rare and has only been reported to occur secondary to periorbital NF. ${ }^{19-21}$ Although the management is similar, there are important clinical and bacteriological differences. Table 1 compares the features of necrotising orbital cellulitis with orbital NF.

In the modern antibiotic era, orbital cellulitis has lost its fearsome reputation as a disease that causes blindness, intracranial complications and death. ${ }^{22}$ Our 2 cases illustrate that orbital cellulitis can be caused by virulent pathogens that can cause atypical severe disease. These pathogens should be suspected when there is rapid progression of orbital signs and severe systemic toxicity and a poor response to appropriate antibiotics. Orbital abscesses must be assumed to be present until proven otherwise. Aggressive surgical drainage of the orbit is an absolute requirement. A failure to improve despite surgery mandates repeated surgery. Without early and aggressive multidisciplinary surgical management there is a high risk of loss of sight and possibly loss of life.

\section{References}

1. Noel LP, Clarke WN, MacDonald N. Clinical management of orbital cellulitis in children. Can J Ophthalmol 1990;25:11-6.

2. Rubin SE, Rubin LG, Zito J, et al. Medical management of orbital subperiosteal abscess in children. J Pediatr Ophthalmol Strabismus 1989;26:21-7.

3. Catalano RA, Smoot CN. Subperiosteal orbital masses in children with orbital cellulitis: time for a re-evaluation? J Pediatr Ophthalmol Strabismus 1990;27:141-2.

4. Lemke BN, Gonnering RS, Weinstein JM. Orbital cellulitis with periosteal elevation. Ophthalmic Plast Reconstr Surg 1987;3:1-7.

5. El-Silimy O. The place of endonasal endoscopy in the treatment of orbital cellulitis. Rhinology 1995;33:93-6.

6. Seah LL, Fu ERY. Acute orbital cellulitis: a review of 17 cases. Ann Acad Med Singapore 1997;26:409-14.

7. Hodges E, Tabbara KF. Orbital cellulitis: review of 23 cases from Saudi Arabia. Br J Ophthalmol 1989;73:205-8. 
8. Bergin DJ, Wright JE. Orbital cellulitis. Br J Ophthalmol 1986;70:174-8.

9. Hofbauer JD, Gordon LK, Palmer J. Acute orbital cellulitis after peribular injection [letter]. Am J Ophthalmol 1994;118:391-2.

10. McLeod SD, Flowers CW, Lopez PF, Marx J, McDonnell PJ. Endophthalmitis and orbital cellulitis after radial keratotomy. Ophthalmology 1995;102:1902-7.

11. Burns SJ, Scott JA, Hiscott PS, Hebbar G, Kamaruddin K, Jalili IK. E. coli panophthalmitis with orbital cellulitis [letter]. Eye 1997;11:436-8.

12. Kingston D, Seal DV. Current hypotheses on synergistic microbial gangrene. Br J Surg 1990;77:260-4.

13. Singh KP, Morris A, Lang SDR, et al. Clinically significant Streptococcus anginosus (Streptococcus milleri) infections: a review of 186 cases. NZ Med J 1988;101:813-6.

14. Rouff KL, Streptococcus anginosus (Streptococcus milleri): the unrecognised pathogen. Clin Microbiol Rev 1988;1:102-8.
15. Mandell GL, Bennett JE, Dollin R. Principles and practice of infectious diseases. 4th ed. New York: Churchill Livingstone, 1995:1861-4.

16. Marrie TJ, Bent JM, West $\mathrm{AB}$, et al. Extensive gas in tissues of the forearm after horsebite. Southern Med J 1979;72:1473-4.

17. Donahue SP, Schwartz G. Preseptal and orbital cellulitis in childhood: a changing microbiologic spectrum. Ophthalmology 1998;105:1902-5.

18. Barone SR, Aiuto LT. Periorbital and orbital cellulitis in the Haemophilus influenzae vaccine era. J Pediatr Ophthalmol Strabismus 1997;35:293-6.

19. Steel A. An unusual case of necrotizing fasciitis. Br J Oral Maxillofacial Surg 1987;25:328-33.

20. Shayegani A, MacFarlane D, Kazim M, et al. Streptococcal gangrene of the eyelids and orbit. Am J Ophthalmol 1995;120:784-92.

21. Holliman RE, Catford GV. Periorbital necrotizing fasciitis with loss of vision. J Infect 1986;13:35-6.

22. Ferguson MP, McNab AA. Current treatment and outcome in orbital cellulitis. Aust NZ J Ophthalmol 1999;27:375-9. 